\title{
RESEARCH
}

Open Access

\section{The incidence of pulmonary thromboembolism in COVID-19 patients admitted to the intensive care unit: a meta- analysis and meta-regression of observational studies}

\author{
Jun Jie $\mathrm{Ng}^{1,2,3^{*}}$ (D), Zhen Chang Liang ${ }^{4}$ and Andrew M. T. L. Choong ${ }^{1,2,3}$
}

\begin{abstract}
Objectives: Coronavirus disease 2019 (COVID-19) infection is associated with a prothrombotic state. We performed a meta-analysis of proportions to estimate the weighted average incidence of pulmonary thromboembolism (PTE) in COVID-19 patients who were admitted to the intensive care unit (ICU).

Methods: We searched various medical databases for relevant studies from 31 December 2019 till 30 September 2020. We included observational studies that reported the incidence of PTE in COVID-19 patients admitted to the ICU. We extracted data related to study characteristics, patient demographics, and the incidence of PTE. Risk of bias was assessed by using the ROBINS-I tool. Statistical analysis was performed with R 3.6.3.

Results: We included 14 studies with a total of 1182 patients in this study. Almost all patients in this meta-analysis received at least prophylactic anticoagulation. The weighted average incidence of PTE was $11.1 \%(95 \% \mathrm{Cl} 7.7 \%$ to $15.7 \%, P^{2}=78 \%$, Cochran's $Q$ test $P<0.01$ ). We performed univariate and multivariate meta-regression, which identified the proportion of males as a significant source of heterogeneity $(P=0.03,95 \% \mathrm{Cl} 0.00$ to -0.09$)$
\end{abstract}

Conclusion: The weighted average incidence of PTE remains high even after prophylactic anticoagulation. PTE is a significant complication of COVID-19 especially in critically ill patients in the ICU.

Keywords: Coronavirus disease 2019, COVID-19, Pulmonary thromboembolism, Intensive care unit, Critical care

\footnotetext{
*Correspondence: jun_jie_ng@nuhs.edu.sg

'Division of Vascular and Endovascular Surgery, Department of Cardiac,

Thoracic and Vascular Surgery, National University Heart Centre, Level 9,

NUHS Tower Block, 1E Kent Ridge Road, Singapore 119228, Singapore

${ }^{2}$ Department of Surgery, Yong Loo Lin School of Medicine, National

University of Singapore, Singapore, Singapore

Full list of author information is available at the end of the article
}

(c) The Author(s). 2021 Open Access This article is licensed under a Creative Commons Attribution 4.0 International License, which permits use, sharing, adaptation, distribution and reproduction in any medium or format, as long as you give appropriate credit to the original author(s) and the source, provide a link to the Creative Commons licence, and indicate if changes were made. The images or other third party material in this article are included in the article's Creative Commons licence, unless indicated otherwise in a credit line to the material. If material is not included in the article's Creative Commons licence and your intended use is not permitted by statutory regulation or exceeds the permitted use, you will need to obtain permission directly from the copyright holder. To view a copy of this licence, visit http://creativecommons.org/licenses/by/4.0/ The Creative Commons Public Domain Dedication waiver (http://creativecommons.org/publicdomain/zero/1.0/) applies to the data made available in this article, unless otherwise stated in a credit line to the data. 


\section{Background}

Since the declaration of a global pandemic by the World Health Organization on 11 March 2020, more than 25 million people have been diagnosed with coronavirus disease 2019 (COVID-19). Among them, over 800,000 people have died [1]. A few distinct themes have emerged as we gradually understand more about the pathophysiology and clinical manifestations of COVID19 infection. One of the more apparent themes is the hypercoagulable, prothrombotic state that critically ill COVID-19 patients are susceptible to [2]. Early studies first reported autopsy findings of micro-thrombus within the pulmonary vasculature of deceased COVID-19 patients [3]. At the same time, other studies started reporting about abnormal coagulation parameters and elevated D-dimer levels in critically ill COVID-19 patients $[4,5]$. On the frontline, physicians treating critically ill COVID-19 patients started noticing an increase in thromboembolic events and line thrombosis [6]. Cognizant of the thromboembolic phenomenon associated with COVID-19, several institutions have published observational studies that reported the incidence of thromboembolic events such as pulmonary thromboembolism (PTE). In this study, we aim to quantitatively synthesize available literature by using meta-analysis of proportions to estimate the weighted average incidence of PTE in critically ill COVID-19 patients admitted to the intensive care unit (ICU).

\section{Methods}

\section{Study protocol}

We conducted this systematic review and meta-analysis following the Cochrane Handbook for Systematic Reviews of Interventions and reported it in accordance with the Preferred Reporting Items for Systematic Reviews and Meta-analyses (PRISMA) guidelines [7, 8]. We formulated the study protocol for this systematic review and meta-analysis in an a priori fashion and published it in PROSPERO (CRD42020188647). Our study protocol is also available as Additional file 1.

\section{Search strategy}

We formulated the search strategy after discussion and consensus by all authors. The search strategy included various combinations and permutations of the following search terms: "coronavirus," "COVID-19," "SARS-CoV2," "2019-nCoV," "thrombus," "thrombo"," "embolus," and "emboli"." Our detailed search protocol is available as Additional file 2. We identified studies by conducting an exhaustive literature search using MEDLINE via PubMed, Embase, and Web of Science. We modified the search syntax for compatibility as required for each database. We only included studies published after 31 December 2019, which corresponds to the date when Chinese officials first reported a cluster of patients diagnosed with pneumonia of unknown cause in Wuhan, Hubei Province, to the World Health Organization [9]. We did not restrict language for the search. After eligible full-text studies were identified, we performed manual backward reference searching to ensure all relevant studies were included. We only included studies that were published in a peer-reviewed journal. We performed a repeat search on 30 September 2020 before submission to ensure no studies were missed.

\section{Eligibility criteria}

We included prospective and retrospective observational studies that reported the incidence of PTE in COVID-19 patients admitted to the ICU for treatment. We excluded individual case reports or case series on PTE in COVID19 patients. We excluded studies that determined the incidence of PTE by reviewing cross-sectional chest imaging regardless of clinical indication. Including these studies may over-estimate the number of patients with PTE as many of these patients with incidental imaging findings of PTE may be clinically asymptomatic. We also excluded studies that had reported the incidence of venous thromboembolism in general without reporting the specific incidence of PTE. Lastly, we excluded studies published in pre-print servers as they are not peerreviewed and might be prone to bias.

\section{Selection of studies and data extraction}

We imported the search items into a commercially available reference manager for deduplication. Following deduplication, two authors (JN and ZL) screened the titles and abstracts for relevant studies. After screening, and obtaining the full-text manuscript of relevant studies, the same two authors reviewed them carefully for inclusion into our systematic review and meta-analysis. Disagreements during abstract and title screening or full-text review were resolved by consensus after discussion with a third author (AC). An author (JN) extracted relevant data from the included studies, and another author (AC) verified the accuracy of the extracted data. We extracted the following variables from the included studies: study first author, study location, study period, study type, study population, study sample size, demographical information (age, gender, body-mass index), comorbidities (diabetes mellitus, hypertension, active malignancy, previous venous thromboembolism), laboratory parameters on admission to ICU (platelet count, D-dimer levels), venous thromboembolism prophylaxis regimens, the proportion of patients on prophylactic or therapeutic anticoagulation, indication for performing PTE imaging, the incidence of PTE, and follow-up period.

\section{Study outcome}

The primary outcome is to estimate and report the weighted average incidence of PTE in critically ill 
COVID-19 patients admitted to the ICU. We considered a positive diagnosis of PTE only if the diagnosis was confirmed by contrast-enhanced computed tomographic imaging of the chest. The secondary outcome is to assess for moderators that could potentially affect the primary outcome.

\section{Risk of bias assessment}

Two authors (JN and ZL) assessed the risk of bias of all included studies using the ROBINS-I tool [10]. Disagreements were resolved by consensus after discussion with a third author (AC). We utilized the ROBINS-I tool as the studies included in the meta-analysis are nonrandomized observational studies. The ROBINS-I tool was explicitly designed to assess the risk of bias in nonrandomized studies in seven domains-bias due to confounding, selection bias, bias in classification of interventions, bias due to deviations from intended interventions, bias due to missing data, bias in the measurement of outcomes, and bias in the selection of reported results. Each domain will be graded to be either at low risk, moderate risk, or high risk of bias. For a domain to be graded low risk, the domain must be comparable to a well-performed randomized trial. For a domain to be graded moderate risk, the domain must be sound for a non-randomized study but cannot be comparable to a well-performed randomized trial. For a domain to be graded serious risk, it means that the domain has some critical issues that need to be addressed. Each included study would be appraised based on each domain's summative grading to deduce the overall risk of bias. If all the domains within a study were graded to have a low risk of bias, the study's overall risk of bias would be low. If any of the domains within a study were graded to have a moderate risk of bias, the study's overall risk of bias would be moderate. If any of the domains within a study were graded to have a serious risk of bias, the study's overall risk of bias would be serious.

\section{Data analysis}

We performed statistical analysis using the meta and metafor packages with $\mathrm{R}$ 3.6.3 ( $\mathrm{R}$ Foundation for Statistical Computing, Vienna, Austria). A frequentist approach was utilized. A meta-analysis of proportions was performed using a random-effects model (DerSimonian and Laird) with logit transformation of observed proportions. The primary outcome was reported as proportions with their respective $95 \%$ confidence intervals (CI). We assessed statistical heterogeneity using the Cochran's $Q$ test and $I^{2}$ statistic. For Cochran's $Q$ test, we used a $P$ value of less than 0.1 to represent significant heterogeneity of intervention effects. For the $I^{2}$ statistic, a value of more than $50 \%$ represented substantial statistical heterogeneity. Initially, as part of our study protocol, we planned to perform further sensitivity analyses such as leave-one-out analysis to explore the sources of study heterogeneity. However, we eventually refrained from performing sensitivity analyses as meta-regression analysis was sufficient to account for the possible moderators that might contribute to statistical heterogeneity. We converted median and interquartile range values to mean and standard deviation for meta-regression analysis using a validated method [11]. We evaluated publication bias with a funnel plot and rank correlation test.

\section{Results \\ Study selection}

A thorough and systematic search was conducted according to the pre-defined search protocol specified in the methods section of this manuscript (Fig. 1). The search yielded a total of 2246 studies, of which 1537 studies remained after deduplication. Following title and abstract screening, we identified 23 studies for full-text review. After completion of the full-text review, we included 14 studies into this systematic review and metaanalysis [12-25].

\section{Risk of bias assessment}

Risk of bias was assessed by using the ROBINS-I tool (Table 1) [10]. A single study was assessed to have a low risk of bias across all domains, and hence deemed to have a low overall risk of bias [14]. Nine studies were considered to have a moderate overall risk of bias, as one or more domains were deemed to be at moderate risk $[13,16,17,19-22,24,25]$. Four studies were considered to have a serious overall risk of bias due to the presence of missing data such as patient comorbidities and ICU characteristics [12, 15, 18, 23].

\section{Characteristics of included studies}

We included 14 studies with a total of 1182 patients into this systematic review and meta-analysis [12-25]. A summary of study characteristics can be seen in Table 2, whereas a summary of patient characteristics can be seen in Table 3. Four studies were conducted in France $[14,16,19,23]$, three in the Netherlands [12, 18, 22], two in Italy [20, 24], two in the UK [13, 25], two in the USA [17, 21], and one in Switzerland [15]. All studies were conducted between February 2020 and April 2020. Only five studies had reported the duration of follow-up, which varied from 7 to 28 days [13, 16, 18, 22, 25].

\section{Indication for ICU admission}

Only four studies had reported their indication for ICU admission [14, 16, 19, 21]. Two studies defined their ICU admission criteria as any patient with respiratory failure $[14,19]$. Helms et al. defined their ICU admission criteria as patients who have acute respiratory distress 


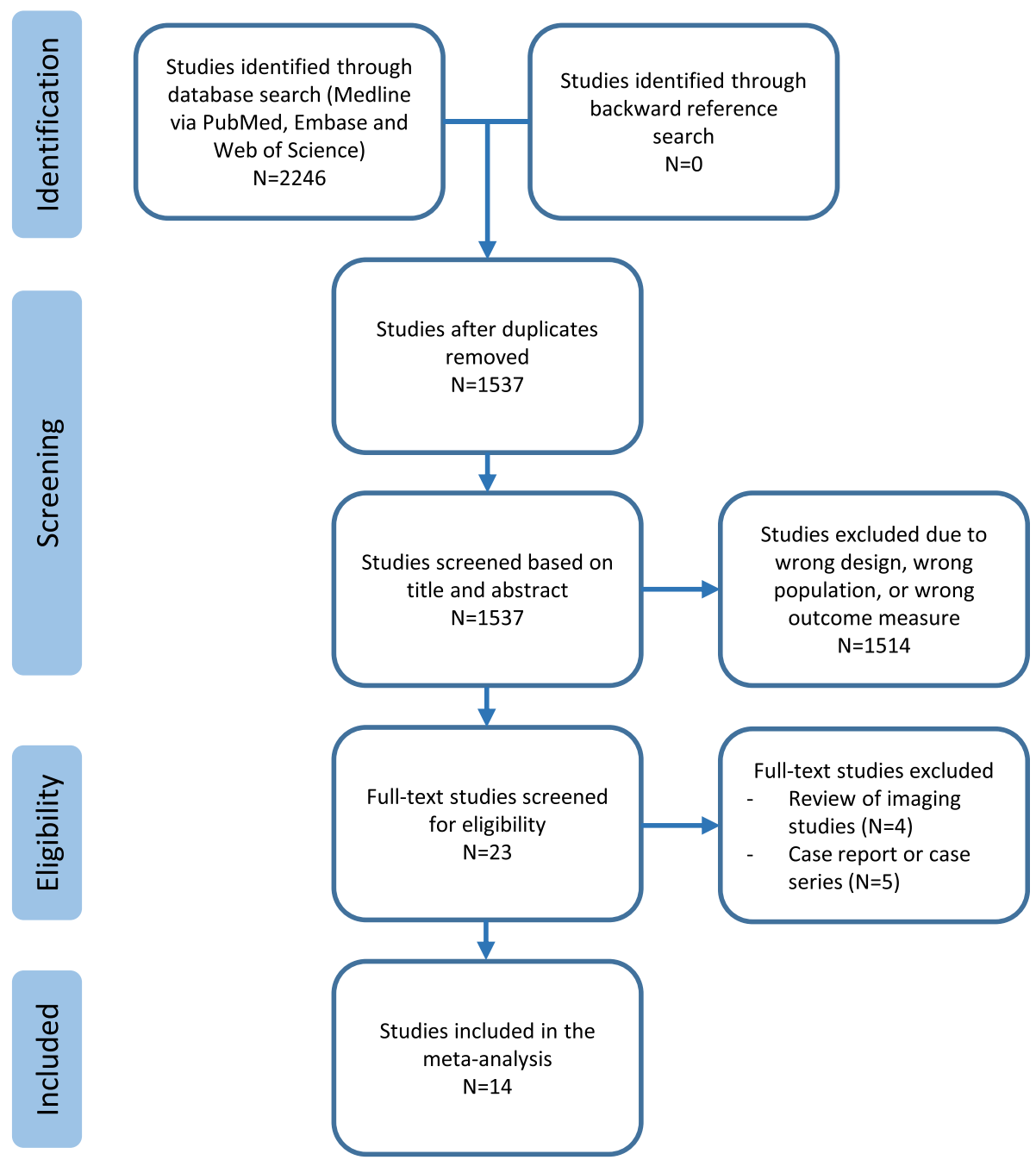

Fig. 1 PRISMA flowchart for study selection

syndrome based on the Berlin 2012 definition [16, 26], whereas the study by Maatman et al. defined their ICU admission criteria as any patient with an oxygen saturation of $94 \%$ or less, respiratory rate of 30 breaths per minute or more, $\mathrm{PaO}_{2} / \mathrm{FiO}_{2}$ ratio of $300 \mathrm{mmHg}$ or less, or if requiring mechanical ventilation [21].

\section{Prophylactic anticoagulation regimen and compliance}

Eleven studies reported using either low-molecularweight heparin (enoxaparin, nadroparin, dalteparin, or unspecified) or unfractionated heparin for venous thromboembolism prophylaxis in varying doses $[13,15$, $16,18-25]$. The majority of studies had also reported information on the proportion of patients receiving therapeutic or prophylactic anticoagulation in ICU. The proportion of patients started on therapeutic anticoagulation in ICU varied from 0 to $69.2 \%$, while the proportion of patients started on prophylactic anticoagulation varied from 30.8 to $100 \%$. Overall, in 10 out of the 11 studies with sufficient information on anticoagulation practices, $100 \%$ of patients received at least prophylactic anticoagulation [13, 14, 16, 18-22, 24, 25]. In the study by Grandmaison et al., $93.1 \%$ of patients received at least prophylactic anticoagulation [15].

\section{Modality and indication for pulmonary thromboembolism imaging \\ Contrast-enhanced computed tomographic scan was the} principal modality used to diagnose PTE in all included studies [12-25]. Eight studies specifically reported the indication for performing PTE imaging [14-16, 19, 20, $22,23,25]$. All the eight studies adopted a selective approach based on the patient's clinical condition to decide if PTE imaging was required. In these studies, PTE imaging was only performed if there was a clinical suspicion of PTE, such as if patients had persistent respiratory failure, deteriorating respiratory function or 
Table 1 Risk of bias assessment with the ROBINS-I tool

\begin{tabular}{|c|c|c|c|c|c|c|c|c|}
\hline Study & Confounding & Selection & $\begin{array}{l}\text { Classification of } \\
\text { interventions }\end{array}$ & $\begin{array}{c}\text { Deviation from } \\
\text { interventions }\end{array}$ & Missing data & $\begin{array}{l}\text { Measurement of } \\
\text { outcomes }\end{array}$ & $\begin{array}{c}\text { Selection of } \\
\text { reported results }\end{array}$ & Overall risk of bias \\
\hline Buen et al.[12] & - & - & $\mathrm{NA}$ & $\mathrm{NA}$ & - & 0 & - & - \\
\hline $\begin{array}{l}\text { Desborough et } \\
\text { al.[13] }\end{array}$ & - & ○ & $\mathrm{NA}$ & NA & - & O & - & ○ \\
\hline Fraissé et al.[14] & - & - & $\mathrm{NA}$ & NA & - & - & - & - \\
\hline $\begin{array}{l}\text { Grandmaison et } \\
\text { al[15] }\end{array}$ & - & ? & NA & NA & $\bullet$ & ○ & ○ & $\bullet$ \\
\hline Helms et al.[16] & ○ & ○ & $\mathrm{NA}$ & NA & ○ & ○ & - & O \\
\hline Hippensteel et al.[17] & ○ & ? & $\mathrm{NA}$ & NA & - & O & - & ○ \\
\hline Klok et al.[18] & - & ○ & NA & NA & • & ○ & - & • \\
\hline Llitjos et al.[19] & - & - & $\mathrm{NA}$ & NA & - & - & - & ○ \\
\hline Lodigiani et al.[20] & - & - & NA & NA & ○ & ○ & - & ○ \\
\hline Maatman et al.[21] & - & 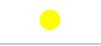 & $\mathrm{NA}$ & NA & - & 0 & - & 0 \\
\hline Middeldorp et al.[22] & - & - & $\mathrm{NA}$ & NA & ○ & - & - & O \\
\hline Poissy et al.[23] & ○ & ○ & NA & $\mathrm{NA}$ & $\bullet$ & ○ & - & - \\
\hline Tavazzi et al.[24] & ○ & - & NA & NA & O & O & ○ & O \\
\hline Thomas et al.[25] & - & ○ & $\mathrm{NA}$ & $\mathrm{NA}$ & O & ○ & - & O \\
\hline
\end{tabular}

Low risk of bias, Moderate risk of bias, Serious risk of bias

NA not applicable

hemodynamic status, or a rapid increase in D-dimer levels.

\section{Primary outcome: incidence of pulmonary thromboembolism}

The reported incidence of PTE ranged from 3.3 to $26.7 \%$. Including all the 14 studies, the weighted average incidence of PTE in COVID-19 patients after admission to the intensive care unit was $11.1 \%$ (95\% CI 7.7 to $15.7 \%, I^{2}$ $=78 \%$, Cochran's $Q$ test $P<0.01$ ) after a random-effects meta-analysis of proportions (Fig. 2) [12-25]. Significant statistical heterogeneity was present as evidenced by high $I^{2}$ value and a Cochran's $Q$ test $P$ value of less than 0.1 .

\section{Meta-regression and moderator assessment}

Meta-regression with a mixed-effects model was performed to examine if the observed heterogeneity could be contributed by possible moderators such as patient or study characteristics (Table 4). Univariate metaregression revealed that the proportion of male patients, platelet count on admission to ICU, and proportion of patients on therapeutic anticoagulation were possible significant moderators. These three significant moderators were added into the multivariable meta-regression model for further analysis. Multivariable meta-regression revealed that the proportion of male patients remained as the only significant moderator in this meta-analysis. A higher proportion of males were associated with a higher incidence of PTE.

\section{Publication bias}

We assessed publication bias by using a funnel plot and the rank correlation test. The funnel plot of all included studies is as shown in Fig. 3. The rank correlation test proved that there was no significant funnel plot asymmetry $(P=0.19)$.

\section{Discussion}

In this meta-analysis, we found that the weighted average incidence of PTE in critically ill COVID-19 patients after admission to the ICU to be $11.1 \%$ (95\% CI 7.7 to 15.7\%). Although other similar meta-analyses have been performed and published in the literature, our study is unique due to several reasons [27]. Firstly, this study was performed specifically to evaluate the risk of pulmonary thromboembolism in COVID-19 patients admitted to the ICU instead of a general hospitalized population. Second, we had strict inclusion criteria and only included studies that started with a cohort of ICU patients. We excluded studies that had identified patients with PTE by examining the reports of all COVID-19 patients who had undergone a computed tomographic scan of the chest. Inclusion of these studies might overestimate the true incidence of symptomatic PTE. Lastly, our study is the only meta-analysis that had included a metaregression analysis to identify and examine the impact of confounder variables on the study effect size. The results from this meta-analysis can be used for several important purposes. First, it can aid in the planning of healthcare resources. As the number of COVID-19 patients 


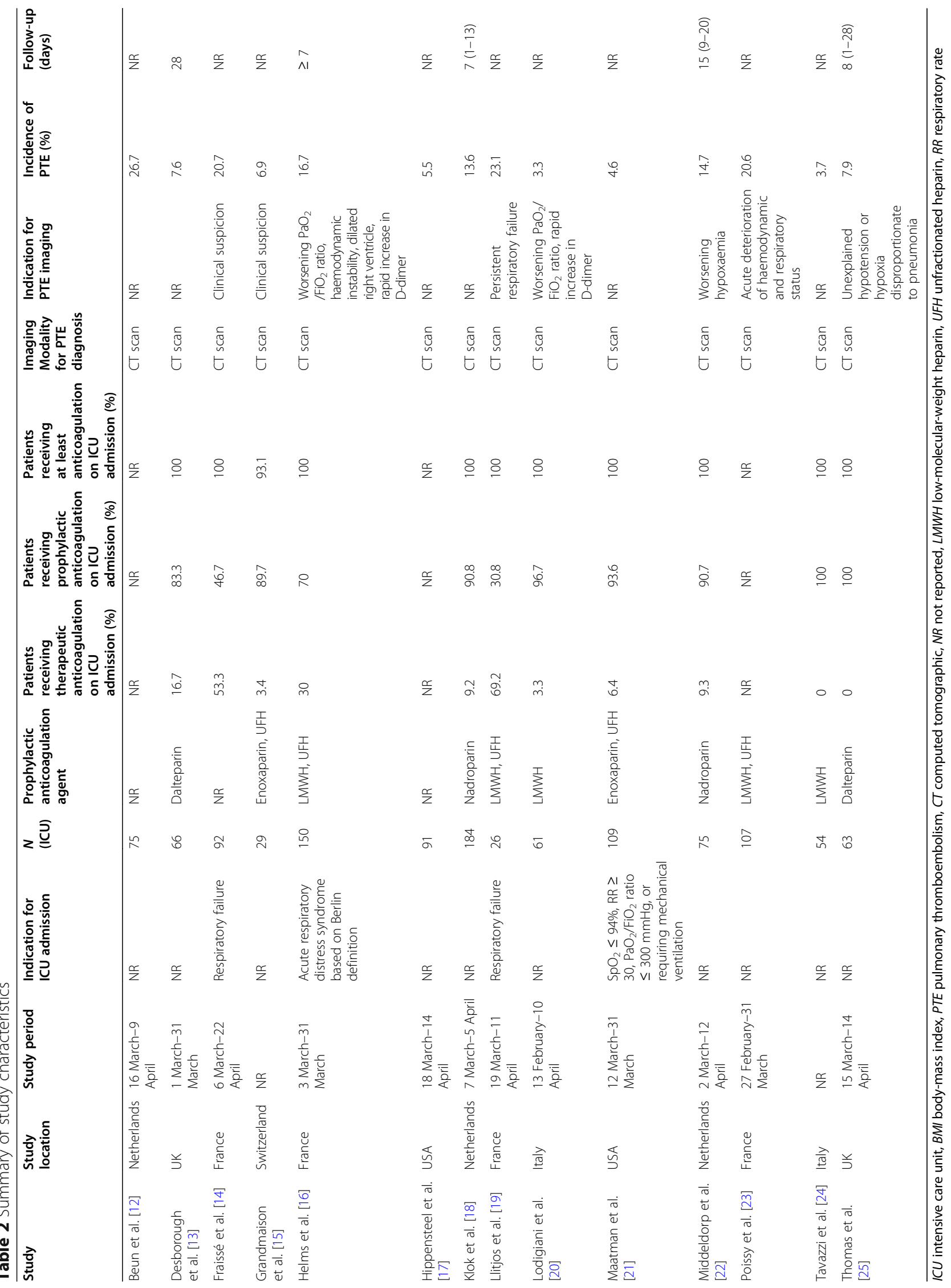




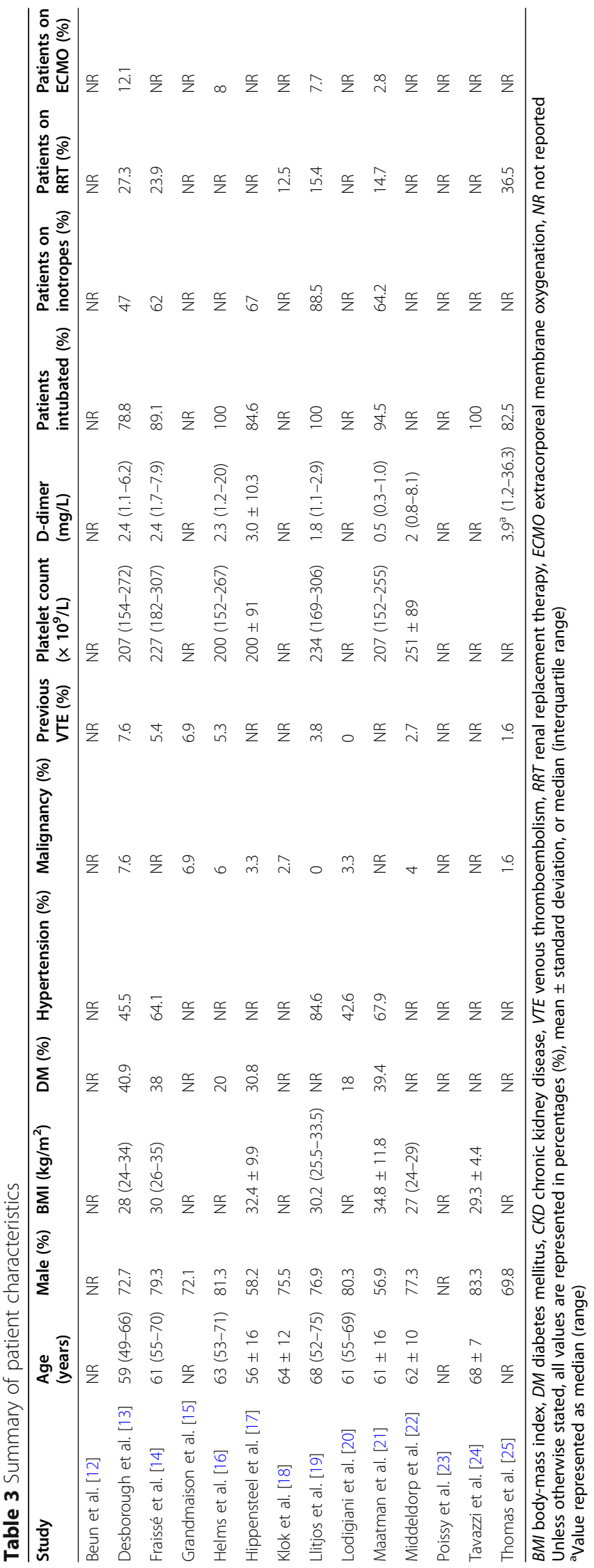




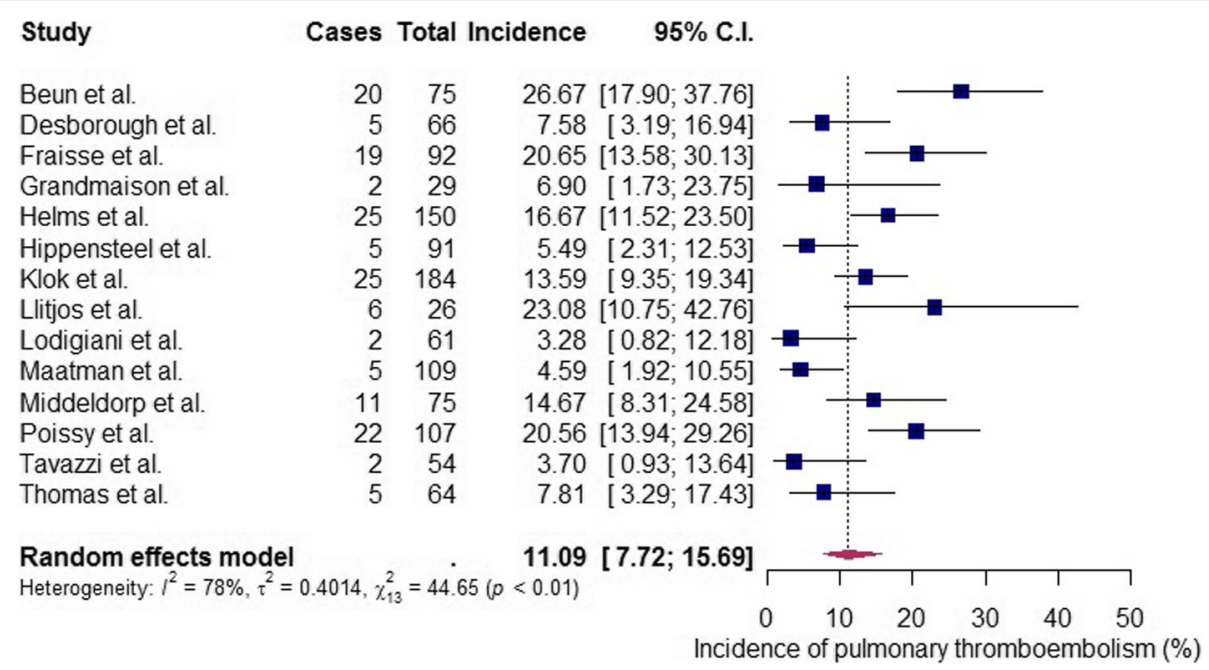

Fig. 2 Forest plot showing the weighted average incidence of pulmonary thromboembolism in critically ill COVID-19 patients admitted to the intensive care unit for treatment

continues to rise, more patients will be admitted to ICU and subsequently diagnosed with PTE. The weighted average incidence of PTE could be used to forecast the need for valuable healthcare resources such as mechanical ventilators as COVID-19 patients with PTE have been shown to require a longer duration of mechanical ventilation [28]. Next, the results from this meta-analysis confirm the thromboembolic risk associated with COVID-19 infection and that a diagnosis of PTE should be considered in any patient with respiratory deterioration. At the same time, patient and family members can be educated on possible complications such as PTE that can arise if admission to ICU is required. Lastly, results from our meta-analysis can also be used to justify the conduct of clinical trials that aim to reduce the risk of thromboembolic events in COVID-19 patients.

We identified the proportion of males to be a significant moderator and significant source of statistical heterogeneity in the incidence of PTE after univariate

Table 4 Meta-regression analysis

\begin{tabular}{|c|c|c|c|c|c|c|c|c|}
\hline \multirow[b]{2}{*}{ Variables } & \multicolumn{4}{|c|}{ Univariate analysis } & \multicolumn{4}{|c|}{ Multivariate analysis } \\
\hline & Coeff & SE & $95 \% \mathrm{Cl}$ & $P$ & Coeff & SE & $95 \% \mathrm{Cl}$ & $P$ \\
\hline Sample size & 0.00 & 0.00 & $-0.01-0.01$ & 0.55 & - & - & - & - \\
\hline Age (years) & 0.06 & 0.08 & $-0.09-0.22$ & 0.40 & - & - & - & - \\
\hline Male gender (\%) & 0.06 & 0.02 & $0.03-0.09$ & $<0.01$ & 0.05 & 0.02 & $0.00-0.09$ & 0.03 \\
\hline Body-mass index $\left(\mathrm{kg} / \mathrm{m}^{2}\right)$ & -0.14 & 0.12 & $-0.37-0.10$ & 0.25 & - & - & - & - \\
\hline Diabetes mellitus (\%) & 0.00 & 0.04 & $-0.08-0.08$ & 0.95 & - & - & - & - \\
\hline Hypertension (\%) & 0.04 & 0.03 & $-0.01-0.09$ & 0.15 & - & - & - & - \\
\hline Active malignancy (\%) & -0.06 & 0.09 & $-0.24-0.13$ & 0.54 & - & - & - & - \\
\hline Previous VTE (\%) & 0.08 & 0.11 & $-0.13-0.28$ & 0.48 & - & - & - & - \\
\hline Platelet count & 0.03 & 0.01 & $0.01-0.05$ & 0.01 & 0.01 & 0.01 & $-0.01-0.02$ & 0.42 \\
\hline D-dimer level & 0.00 & 0.00 & $-0.00-0.01$ & 0.44 & - & - & - & - \\
\hline $\begin{array}{l}\text { Patients on therapeutic } \\
\text { anticoagulation (\%) }\end{array}$ & 0.02 & 0.01 & $0.01-0.03$ & $<0.01$ & 0.01 & 0.01 & $-0.01-0.03$ & 0.24 \\
\hline Patients intubated (\%) & 0.03 & 0.04 & $-0.04-0.10$ & 0.43 & - & - & - & - \\
\hline Patients on inotropes (\%) & 0.03 & 0.03 & $-0.03-0.09$ & 0.36 & - & - & - & - \\
\hline Patients on RRT (\%) & -0.02 & 0.04 & $-0.09-0.06$ & 0.66 & - & - & - & - \\
\hline Patients on ECMO (\%) & 0.07 & 0.15 & $-0.21-0.35$ & 0.63 & - & - & - & - \\
\hline
\end{tabular}

$B M I$ body mass index, Coeff coefficient, $C I$ confidence interval, ECMO extracorporeal membrane oxygenation, $R R T$ renal replacement therapy, SE standard error, $V T E$ venous thromboembolism 


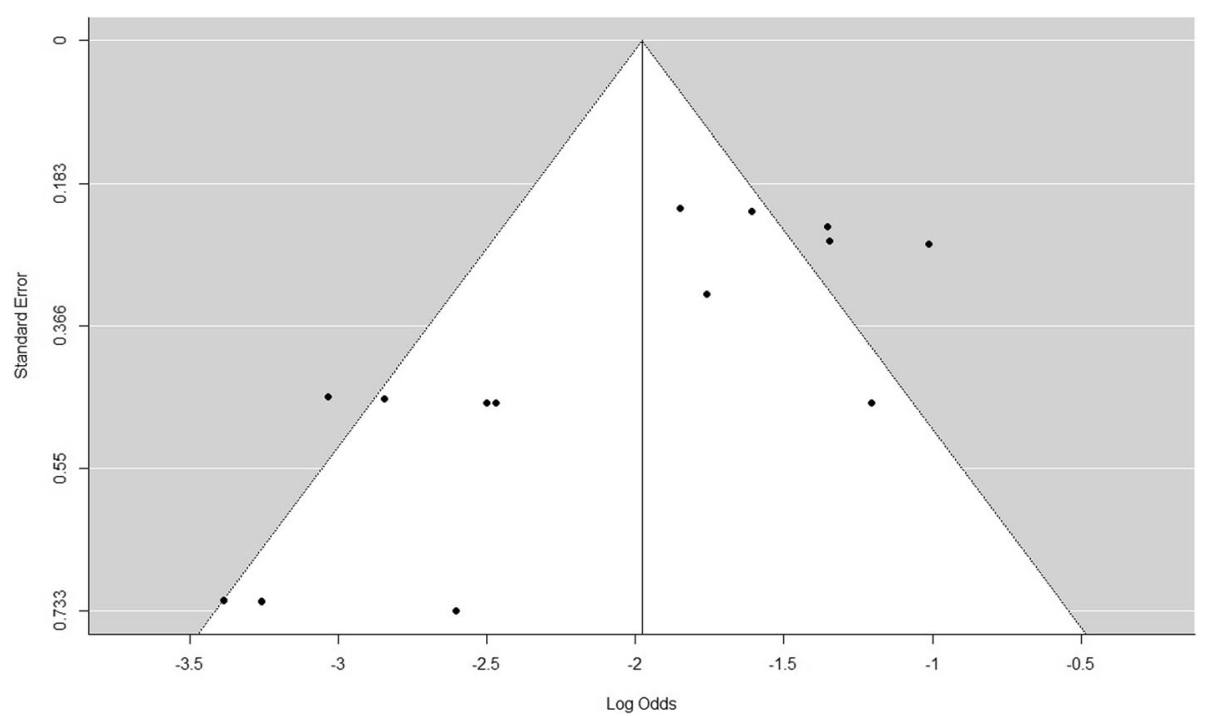

Fig. 3 Funnel plot for assessment of publication bias

and multivariate meta-regression analysis. Our analysis shows that studies with a higher proportion of male patients had a higher incidence of reported PTE. This observed phenomenon can be corroborated by several studies performed in the past that have demonstrated a higher risk of first episode or recurrent venous thromboembolism in males than females [29]. Several hypotheses such as genetic variations or differences in environmental factors have been suggested to account for the differential risks of venous thromboembolism, but the evidence remains unclear [30].

The significant statistical heterogeneity of our primary outcome could also be explained by other moderators that were not included in our meta-regression analysis. One possibility that cannot be easily analyzed or accounted for would be how study location and study period can affect the incidence of PTE. Studies by Lodigiani et al. and Tavazzi et al., which were both conducted in Italy, had reported relatively low PTE incidences of $3.3 \%$ and $3.7 \%$ respectively compared to studies conducted outside of Italy [20,24]. We postulate several possible reasons for the observed geographical disparity in reported PTE incidences. First, Lodigiani et al. had reported that only $13.1 \%$ of their study population had PE imaging performed [20]. Compared to other studies included in this meta-analysis, this seemed to be considerably lower. As such, PTE could have been underdiagnosed. On the contrary, other studies with more widespread PTE imaging may have diagnosed many patients with subclinical or asymptomatic PTE. Several retrospective studies reviewed CT scans performed COVID-19 patients regardless of clinical context had reported radiological findings of PTE in up to 50\% of patients $[28,31]$. Undoubtedly, most PTE findings in these radiological studies may be clinically insignificant. Furthermore, resource constraints secondary to the COVID-19 pandemic might contribute to the possible geographical disparity in PTE incidence. The studies by Lodigiani et al. and Tavazzi et al. were conducted in the Lombardy region of Italy, which had the most number of COVID-19 cases and the highest case fatality rate in Italy [32]. As such, the institutions at which the studies were conducted might have faced possible resource constraints that may have inevitably led to a more selective or conservative approach to PTE imaging and diagnosis [33]. Lastly, other moderators such as follow-up duration might have also contributed to the statistical heterogeneity, but cannot be adequately evaluated due to missing data.

Our study also provides some insight into the various prophylactic anticoagulation regimens adopted by different institutions. Interestingly, two studies conducted in the Netherlands had doubled the doses of their anticoagulation regimens around late March and early April $[18,22]$. This is most likely due to an increased awareness of the thromboembolic manifestations of COVID19. Ten studies had also reported that $100 \%$ of their study population had received at least prophylactic anticoagulation [13, 14, 16, 18-22, 24, 25]. However, despite a high degree of compliance to prophylactic anticoagulation, a considerable proportion of critically ill COVID-19 patients still developed PTE. Several studies have suggested an intermediate dose or therapeutic anticoagulation regimen for this group of patients due to this phenomenon [34, 35]. Several institutions worldwide are currently planning or have begun recruiting for clinical trials to evaluate the effects of higher dose anticoagulation for critically ill COVID-19 patients [36]. 
There are several limitations to our meta-analysis. First, we could not fully account for the differences in study population and study design. Many of the included studies did not report essential information such as the indication for ICU admission, exact type and dosages of anticoagulation therapy used, disease severity, and use of adjunctive therapies such as rehabilitation and follow-up duration. Due to significant missing data, 4 out of the 14 included studies were deemed to have high overall risk of bias. Next, we were unable to assess the effect of anticoagulation use on the incidence of clinically relevant adverse events such as minor or major hemorrhage as the included studies did not report them. Such information is vital as clinicians routinely need to decide between anticoagulation use and risk of hemorrhage. Lastly, we were also unable to assess the effect of PTE incidence on mortality, as most studies did not report mortality outcomes.

\section{Conclusion}

In conclusion, the weighted average incidence of PTE in critically ill COVID-19 patients admitted to the ICU for treatment was $11.1 \%$, even though almost all patients received at least prophylactic doses of anticoagulation therapy. Although there was significant heterogeneity in our meta-analysis, we identified the proportion of male patients as a significant moderator and contributor of heterogeneity. Most included studies have moderate to serious risks of bias, and results should be interpreted with caution. Clinicians should be aware that PTE can occur in a significant proportion of COVID-19 patients receiving ICU care despite adequate prophylactic anticoagulation and should investigate further should clinical suspicion arises. Moving ahead, more studies are also needed to determine the optimal anticoagulation strategy in critically ill COVID-19 patients.

\section{Supplementary Information}

The online version contains supplementary material available at https://doi. org/10.1186/s40560-021-00535-x.

Additional file 1. Study protocol.

Additional file 2. Search strategy and keywords.

\section{Abbreviations}

COVID-19: Coronavirus disease 2019; PTE: Pulmonary thromboembolism; ICU: Intensive care unit; $\mathrm{Cl}$ : Confidence intervals

\section{Acknowledgements}

Not applicable

\section{Authors' contributions}

Jun Jie $\mathrm{Ng}(\mathrm{JN})$ and Zhen Chang Liang (ZL) contributed both contributed equally to the conception, design of this study; acquisition, analysis, and interpretation of data. JN drafted the initial manuscript. Andrew MTL Choong (AC) contributed to the design of this study; acquisition and interpretation of data; and critically revised the manuscript. All authors have approved the submitted version of this manuscript. The corresponding author (JN) attests that all listed authors meet authorship criteria and that no others meeting.

Funding

The authors declare no source of funding was utilized in this study.

\section{Availability of data and materials}

The datasets used and/or analyzed during the current study are available from the corresponding author on reasonable request.

Ethics approval and consent to participate

Ethics approval was not required for this study as it does not contain any form of individual patient data.

Consent for publication

Not applicable

\section{Competing interests}

The authors declare that they have no competing interests.

\section{Author details}

${ }^{1}$ Division of Vascular and Endovascular Surgery, Department of Cardiac, Thoracic and Vascular Surgery, National University Heart Centre, Level 9, NUHS Tower Block, 1E Kent Ridge Road, Singapore 119228, Singapore. Department of Surgery, Yong Loo Lin School of Medicine, National University of Singapore, Singapore, Singapore. ${ }^{3}$ SingVaSC, Singapore Vascular Surgical Collaborative, Singapore, Singapore. ${ }^{4}$ Department of Orthopaedic Surgery, National University Hospital, Singapore, Singapore.

Received: 7 November 2020 Accepted: 3 February 2021

Published online: 22 February 2021

\section{References}

1. World Health Organization. COVID-19 situation reports. 2020, https://www. who.int/emergencies/diseases/novel-coronavirus-2019/situation-reports/. Accessed 1 Sept 2020.

2. Castelli R, Gidaro A. Abnormal hemostatic parameters and risk of thromboembolism among patients with COVID-19 infection. J Hematol. 2020;9(1-2):1-4

3. Yao XH, Li TY, He ZC, et al. [A pathological report of three COVID-19 cases by minimally invasive autopsies]. Zhonghua Bing Li Xue Za Zhi. [published online: March 15, 2020]. 10.3760/cma.j.cn112151-2020031200193

4. Han H, Yang L, Liu R, et al. Prominent changes in blood coagulation of patients with SARS-CoV-2 infection. Clin Chem Lab Med. [published online: March 16, 2020]. 10.1515/cclm-2020-0188

5. Tang N, Li D, Wang X, Sun Z. Abnormal coagulation parameters are associated with poor prognosis in patients with novel coronavirus pneumonia. J Thromb Haemost. 2020;18(4):844-7.

6. Poggiali E, Bastoni D, loannilli E, Vercelli A, Magnacavallo A. Deep vein thrombosis and pulmonary embolism: two complications of COVID-19 pneumonia. Eur J Case Rep Intern Med. 2020;7(5):001646.

7. Cochrane Training: Cochrane Handbook for Systematic Reviews of Interventions. 2020, https://training.cochrane.org/handbook. Accessed 10 May 2020.

8. Moher D, Liberati A, Tetzlaff J, et al. Preferred reporting items for systematic reviews and meta-analyses: the PRISMA statement. BMJ. 2009;339:b2535.

9. World Health Organization: WHO Timeline - COVID-19. 2020, https://www. who.int/news-room/detail/27-04-2020-who-timeline\%2D\%2D-covid-19. Accessed 1 Sept 2020.

10. Sterne JA, Hernán MA, Reeves BC, et al. ROBINS-l: a tool for assessing risk of bias in non-randomised studies of interventions. BMJ. 2016:355:i4919.

11. Wan X, Wang W, Liu J, Tong T. Estimating the sample mean and standard deviation from the sample size, median, range and/or interquartile range. BMC Med Res Methodol. 2014;14:135.

12. Beun R, Kusadasi N, Sikma M, Westerink J, Huisman A. Thromboembolic events and apparent heparin resistance in patients infected with SARS-CoV2. Int J Lab Hematol. 2020;42(Suppl 1):19-20.

13. Desborough MJR, Doyle AJ, Griffiths A, Retter A, Breen KA, Hunt BJ. Image-proven thromboembolism in patients with severe COVID-19 in 
a tertiary critical care unit in the United Kingdom. Thromb Res. 2020; 193:1-4.

14. Fraissé M, Logre E, Pajot O, Mentec H, Plantefève G, Contou D. Thrombotic and hemorrhagic events in critically ill COVID-19 patients: a French monocenter retrospective study. Crit Care. 2020;24(1):275.

15. Grandmaison G, Andrey A, Périard D, et al. Systematic Screening for Venous Thromboembolic Events in COVID-19 Pneumonia. TH Open. 2020;4(2):e113-5.

16. Helms J, Tacquard C, Severac F, et al. High risk of thrombosis in patients with severe SARS-CoV-2 infection: a multicenter prospective cohort study. Intensive Care Med. [published online: May 04, 2020]. 10.1007/s00134-02006062-x

17. Hippensteel JA, Burnham EL, Jolley SE. Prevalence of venous thromboembolism in critically ill patients with COVID-19. Br J Haematol. 2020;190(3):e134-7.

18. Klok FA, Kruip MJHA, van der Meer NJM, et al. Incidence of thrombotic complications in critically ill ICU patients with COVID-19. Thromb Res. [published online: April 10, 2020]. 10.1016/j.thromres.2020.04.013

19. Llitjos JF, Leclerc M, Chochois $C$, et al. High incidence of venous thromboembolic events in anticoagulated severe COVID-19 patients. J Thromb Haemost. [published online: April 22, 2020]. 10.1111/jth.14869

20. Lodigiani $\mathrm{C}$, lapichino $\mathrm{G}$, Carenzo L, et al. Venous and arterial thromboembolic complications in COVID-19 patients admitted to an academic hospital in Milan, Italy. Thromb Res. [published online: April 23, 2020]. 10.1016/j.thromres.2020.04.024

21. Maatman TK, Jalali F, Feizpour C, et al. Routine venous thromboembolism prophylaxis may be inadequate in the hypercoagulable state of severe coronavirus disease 2019. Crit Care Med. 2020;48(9):e783-90.

22. Middeldorp S, Coppens M, van Haaps TF, et al. Incidence of venous thromboembolism in hospitalized patients with COVID-19. J Thromb Haemost. [published online: May 05, 2020]. 10.1111/jth.14888

23. Poissy J, Goutay J, Caplan M, et al. Pulmonary embolism in COVID-19 patients: awareness of an increased prevalence. Circulation. [published online: April 24, 2020]. 10.1161/CIRCULATIONAHA.120.047430

24. Tavazzi G, Civardi L, Caneva L, Mongodi S, Mojoli F. Thrombotic events in SARS-CoV-2 patients: an urgent call for ultrasound screening. Intensive Care Med. [published online: April 22, 2020]. 10. 1007/s00134-020-06040-3

25. Thomas W, Varley J, Johnston A, et al. Thrombotic complications of patients admitted to intensive care with COVID-19 at a teaching hospital in the United Kingdom. Thromb Res. [published online: April 25, 2020]. 10.1016/j. thromres.2020.04.028

26. The ARDS Definition Task Force* ${ }^{*}$. Acute respiratory distress syndrome: the Berlin definition. JAMA. 2012;307(23):2526-33.

27. Porfidia A, Valeriani E, Pola R, Porreca E, Rutjes AWS, Di Nisio M. Venous thromboembolism in patients with COVID-19: Systematic review and meta-analysis [published online: Aug 12, 2020]. Thromb Res. 2020;196:67-74.

28. Grillet F, Behr J, Calame P, Aubry S, Delabrousse E. Acute pulmonary embolism associated with COVID-19 pneumonia detected by pulmonary CT angiography. Radiology. [published online: April 23, 2020]. 10.1148/radiol. 2020201544

29. Douketis J, Tosetto A, Marcucci M, et al. Risk of recurrence after venous thromboembolism in men and women: patient level meta-analysis. BMJ. 2011;342:d813.

30. Roach REJ, Cannegieter SC, Lijfering WM. Differential risks in men and women for first and recurrent venous thrombosis: the role of genes and environment. J Thromb Haemost. 2014;12:1593-600.

31. Leonard-Lorant I, Delabranche X, Severac F, et al. Acute pulmonary embolism in COVID-19 patients on CT angiography and relationship to Ddimer levels. Radiology. [published online: April 23, 2020]. 10.1148/radiol. 2020201561

32. Sebastiani G, Massa M, Riboli E. Covid-19 epidemic in Italy: evolution, projections and impact of government measures. Eur J Epidemiol. 2020; 35(4):341-5.

33. Grasselli G, Pesenti A, Cecconi M. Critical care utilization for the COVID-19 outbreak in Lombardy, Italy: early experience and forecast during an emergency response. JAMA. [published online: March 13, 2020]. 10.1001/ jama.2020.4031
34. Song JC, Wang G, Zhang W, Zhang Y, Li WQ, Zhou Z. Chinese expert consensus on diagnosis and treatment of coagulation dysfunction in COVID-19. Mil Med Res. 2020;7(1):19.

35. Thachil J, Tang N, Gando S, et al. ISTH interim quidance on recognition and management of coagulopathy in COVID-19. J Thromb Haemost. 2020;18(5): 1023-6.

36. U.S. National Library of Medicine ClinicalTrials.gov: Intermediate or prophylactic-dose anticoagulation for venous or arterial thromboembolism in severe COVID-19 (IMPROVE) (NCT04367831). 2020, https://clinicaltrials. gov/ct2/show/NCT04367831. Accessed 14 May 2020.

\section{Publisher's Note}

Springer Nature remains neutral with regard to jurisdictional claims in published maps and institutional affiliations.
Ready to submit your research? Choose BMC and benefit from:

- fast, convenient online submission

- thorough peer review by experienced researchers in your field

- rapid publication on acceptance

- support for research data, including large and complex data types

- gold Open Access which fosters wider collaboration and increased citations

- maximum visibility for your research: over $100 \mathrm{M}$ website views per year

At $\mathrm{BMC}$, research is always in progress.

Learn more biomedcentral.com/submissions 